\title{
A New Subclass of Meromorphic Univalent Functions with Positive Coefficients Defined by Linear Operator
}

\author{
${ }^{1}$ Sarikonda Sreelakshmi, ${ }^{2}$ Rajkumar N.Ingle, ${ }^{3}$ P.Thirupathi Reddy \\ ${ }^{1}$ PGT in Maths, Telangana Social Welfare Residential School/Junior College (Girls) \\ Elkathurthy, Dist. Warangal (Urban), Telangana - 505476 \\ ${ }^{2}$ Department of Mathematics, \\ Bahirji Smarak Mahavidyalay, Bashmathnagar, Dist.HINGOLI(M.S.) \\ ${ }^{3}$ Department of Mathematics , Kakatiya University, Warangal-506009 \\ Telangana.
}

\begin{abstract}
In this paper, we introduce and study new class $\Sigma_{p}(\alpha, \beta)$ of meromorphically univalent functions defined in $E=\{z: z \in C$ and $0<|z|<1\}$. We obtain coefficients inequalities distortion theorems, radius of convexity, Closure theorems and modified Hadamard products. Finally we obtain application involving an integral transforms and neighbourhood properties for the class $\sum_{p}(\alpha, \beta)$.
\end{abstract}

Keywords and Phrases : Meromorphic, distortion,Hadamard product and neighbourhood.

2010 Mathematical Mubject Classification : 30C45.

\section{INTRODUCTION}

Let $\Sigma^{*}$ denote the class of meromorphic functions of the form

$$
f(z)=\frac{1}{z}+\sum_{k=1}^{\infty} a_{k} z^{k},\left(a_{k} \geq 0\right)
$$

which are analytic in the punctured unit disc $E=\{z: z \in C$ and $0<|z|<1\}$

Let $g(z) \in \Sigma^{*}$, be given by

$$
g(z)=\frac{1}{z}+\sum_{k=1}^{\infty} b_{k} z^{k}
$$

Then the Hadamard product (or convolution) of $f(\mathrm{z})$ and $\mathrm{g}(\mathrm{z})$ is given by

$$
(f * g)(z)=\frac{1}{z}+\sum_{k=1}^{\infty} a_{k} b_{k} z^{k}
$$

A function $f(z) \in \Sigma^{*}$ is meromorphically star like of order $\alpha(0 \leq \alpha<1)$ if

$$
-\operatorname{Re}\left\{\frac{z f^{\prime}(z)}{f(z)}\right\}>\alpha,(z \in E)
$$

The class of all such functions is denoted by $\Sigma^{*}(\alpha)$. A function $f \in \Sigma^{*}$ is meromorphically convex of order $\alpha(0 \leq \alpha<1)$ if 
- $\operatorname{Re}\left\{1+\frac{z f^{\prime \prime}(z)}{f^{\prime}(z)}\right\}>\alpha,(z \in E)$

The class of such functions is denoted by $\sum_{k}{ }_{k}^{*}(\alpha)$. The classes $\sum^{*}(\alpha)$ and $\sum_{k}{ }_{k}^{*}(\alpha)$ were introduced and studied by Pommerenke [6], Miller [4], Mogra et al [5] and others.

For a function $f(z) \in \Sigma^{*}$, Frasin and Darus [2] defined an operator $I^{n}: \Sigma^{*} \rightarrow \Sigma^{*}$ as follows.

$$
\begin{aligned}
& I^{0} f(z)=f(z) \\
& I^{1} f(z)=z f^{\prime}(z)+\frac{2}{z} \\
& I^{2} f(z)=z\left(I^{1} f(z)\right)^{\prime}+\frac{2}{z}
\end{aligned}
$$

and for $n \in N=\{1,2 \ldots \ldots$.$\} , we have$

$$
\begin{aligned}
& I^{n} f(z)=z\left(I^{n-1} f(z)\right)^{\prime}+\frac{2}{z} \\
& \quad=\frac{1}{z}+\sum_{k=1}^{\infty} k^{n} a_{k} z^{k},\left(n \in N_{0}=N \cup\{0\}, z \in E\right)
\end{aligned}
$$

Now, we define a new subclass $\Sigma_{p}(\alpha, \beta)$ of $\Sigma$.

Definition 1 : For $-1 \leq \alpha<1$, and $\beta \geq 1$, we let $\Sigma_{p}(\alpha, \beta)$ be the subclass of $\Sigma$ consisting of functions of the form (1.6) and satisfying the analytic criterion

$$
\operatorname{Re}\left\{\frac{I^{n+1} f(z)}{I^{n} f(z)}-\alpha\right\}>\beta\left|\frac{I^{n+1} f(z)}{I^{n} f(z)}-1\right|
$$

were $I^{n} f(z)$ in given by (1.6).

\section{COEFFICIENT INEQUALITY}

Theorem 2.1. A function $f(\mathrm{z})$ of the form (1.6) is in $\Sigma_{p}(\alpha, \beta)$ if

$$
\sum_{k=1}^{\infty} k^{n}[k(1+\beta)-(\alpha+\beta)]\left|a_{k}\right| \leq(1-\alpha),-1 \leq \alpha<1 \text {, and } \beta \geq 1 \text {. }
$$

Proof. It suffices to show that

We have

$$
\beta\left|\frac{I^{n+1} f(z)}{I^{n} f(z)}-1\right|-\operatorname{Re}\left\{\frac{I^{n+1} f(z)}{I^{n} f(z)}-1\right\} \leq 1-\alpha .
$$

$$
\begin{array}{r}
\beta\left|\frac{I^{n+1} f(z)}{I^{n} f(z)}-1\right|-\operatorname{Re}\left\{\frac{I^{n+1} f(z)}{I^{n} f(z)}-1\right\} \\
\leq(1+\beta)\left|\frac{I^{n+1} f(z)}{I^{n} f(z)}-1\right| \\
\leq \frac{(1+\beta) \sum_{k=1}^{\infty} k^{n}(k-1)\left|a_{k}\right|\left|z^{k}\right|}{\frac{1}{|z|}-\sum_{k=1}^{\infty} k^{n}\left|a_{k}\right|\left|z^{k}\right|}
\end{array}
$$

Letting $\mathrm{z} \rightarrow 1$ along the real axis, we obtain 


$$
\frac{(1+\beta) \sum_{k=1}^{\infty} k^{n}(k-1)\left|a_{k}\right|}{1-\sum_{k=1}^{\infty} k^{n}\left|a_{k}\right|}
$$

This last expression is bounded by $1-\alpha$ if

$$
\sum_{k=1}^{\infty} k^{n}[k(1+\beta)-(\alpha+\beta)]\left|a_{k}\right| \leq(1-\alpha) \text {. }
$$

Corollary 2.1. Let the function $f(\mathrm{z})$ defined by (1.6) be in the class $\Sigma_{p}(\alpha, \beta)$ then

$$
a_{k} \leq \frac{(1-\alpha)}{\sum_{k=1}^{\infty} k^{n}[k(1+\beta)-(\alpha+\beta)]}, k \geq 1 .
$$

Equality holds for the functions of the form

$$
f_{k}(z)=\frac{1}{z}+\frac{(1-\alpha)}{k^{n}[k(1+\beta)-(\alpha+\beta)]} z^{k}
$$

\section{DISTORTION THEOREMS}

Theorem 3.1. Let the function $\mathrm{f}(\mathrm{z})$ defined by (1.6) be in the class $\sum_{p}(\alpha, \beta)$.

Then for $0<|z|=r<1$,

$$
\frac{1}{r}-r \leq|f(z)| \leq \frac{1}{r}+r
$$

with equality for the function $f(z)=\frac{1}{z}+z$,

Proof. Suppose $f(z)$ is in $\Sigma_{p}(\alpha, \beta)$. In view of Theorem 2.1, we have

$$
(1-\alpha) \sum_{k=1}^{\infty} a_{k} \leq k^{n}[k(1+\beta)-(\alpha+\beta)] \leq(1-\alpha)
$$

which evidently yields $\sum_{k=1}^{\infty} a_{k} \leq 1$ consequently, we obtain

$$
\begin{aligned}
|f(z)|=\left|\frac{1}{z}+\sum_{k=1}^{\infty} a_{k} z^{k}\right| & \leq\left|\frac{1}{z}\right|+\sum_{k=1}^{\infty} a_{k}|z|^{k} \\
& \leq \frac{1}{r}+r \sum_{k=1}^{\infty} a_{k} \\
& \leq \frac{1}{r}+r
\end{aligned}
$$

Also,

$$
\begin{aligned}
|f(z)|=\left|\frac{1}{z}+\sum_{k=1}^{\infty} a_{k} z^{k}\right| & \geq\left|\frac{1}{z}\right|-\sum_{k=1}^{\infty} a_{k}|z|^{k} \\
& \geq \frac{1}{r}-r \sum_{k=1}^{\infty} a_{k}
\end{aligned}
$$




$$
\geq \frac{1}{r}-r
$$

Hence the results (3.1) follow.

Theorem 3.2. Let the function $\mathrm{f}(\mathrm{z})$ defined by (1.6) be in the class $\Sigma_{p}(\alpha, \beta)$. Then for $0<|z|=r<1$,

$$
\frac{1}{r^{2}}-1 \leq\left|f^{1}(z)\right| \leq \frac{1}{r^{2}}+1
$$

The result is sharp, the external function being of the form (2.2)

Proof : From Theorem 2.1, we have

$$
(1-\alpha) \sum_{k=1}^{\infty} n a_{k} \leq k^{n}[k(1+\beta)-(\alpha+\beta)] \leq(1-\alpha)
$$

which evidently yields

$$
\sum_{k=1}^{\infty} k a_{k} \leq 1
$$

Consequently, we obtain

$$
\begin{aligned}
\left|f^{\prime}(z)\right| & \leq \frac{1}{r^{2}}+\sum_{k=1}^{\infty} k a_{k} r^{k-1} \\
& \leq \frac{1}{r^{2}}+\sum_{k=1}^{\infty} k a_{k} \\
& \leq \frac{1}{r^{2}}+1
\end{aligned}
$$

Also

$$
\begin{aligned}
\left|f^{\prime}(z)\right| & \geq \frac{1}{r^{2}}-\sum_{k=1}^{\infty} k a_{k} r^{k-1} \\
& \geq \frac{1}{r^{2}}-\sum_{k=1}^{\infty} k a_{k} \\
& \geq \frac{1}{r^{2}}+1
\end{aligned}
$$

This completes the proof.

\section{CLASS PRESERVING INTEGRAL OPERATORS}

In this section we consider the class preserving integral operators of the form (1.6)

Theorem 4.1. Let the function $f(\mathrm{z})$ be define by (1.6) be in the class $\sum_{p}(\alpha, \beta)$. Then the integral operator

$$
\begin{aligned}
& F(z)=c z^{-c-1} \int_{0}^{z} t^{c} f(t) d t=\frac{1}{z}+\sum_{k=1}^{\infty} \frac{c}{c+k+1} a_{k} z^{k}, c>0 \text { is in } \sum_{p}(\delta, \beta), \text { where } \\
& \delta \leq \frac{(c+k+1)[k(1+\beta)-(\alpha+\beta)]-[k(1+\beta)-\beta] c(1-\alpha)}{(c+k+1)[k(1+\beta)-(\alpha+\beta)]+c(1-\alpha)}
\end{aligned}
$$

The result is sharp for function defined in (2.3).

Proof: Suppose $f(z) \in \sum_{p}(\alpha, \beta)$, we have 


$$
F(z)=c z^{-c-1} \int_{0}^{z} t^{c} f(t) d t=\frac{1}{z}+\sum_{k=1}^{\infty} \frac{c}{c+k+1} a_{k} z^{k}, c>0
$$

It is sufficient to show that

$$
\sum_{k=1}^{\infty} \frac{k^{n}[k(1+\beta)-(\delta+\beta)]}{1-\delta} \frac{c}{c+k+1} a_{k} \leq 1
$$

Since $f(z)$ is in $\sum_{p}(\alpha, \beta)$, we have

$$
\sum_{k=1}^{\infty} \frac{k^{n}[k(1+\beta)-(\alpha+\beta)]}{1-\alpha} \leq 1
$$

This (4.3) will be satisfied if

$$
\frac{[k(1+\beta)-(\delta+\beta)]}{1-\delta} \frac{c}{c+k+1} \leq \frac{[k(1+\beta)-(\alpha+\beta)]}{1-\alpha}
$$

Solving for $\delta$, we obtain

$$
\delta \leq \frac{(c+k+1)[k(1+\beta)-(\alpha+\beta)]-[k(1+\beta)-\beta] c(1-\alpha)}{(c+k+1)[k(1+\beta)-(\alpha+\beta)]+c(1-\alpha)}=F(k)
$$

A simple computation will show that $F(k)$ is increasing and $F(k) \geq F(1)$.

\section{RADII OF CONVEXITY}

Theorem 5.1. Let the function $f(z) \in \sum_{p}(\alpha, \beta)$. Then $\mathrm{f}(\mathrm{z})$ is Meromorphically convex of order $\delta(0 \leq \delta<1)$ in $0<|z|<r$, where

$$
\gamma(\alpha, \beta, \delta)=\inf _{n \geq 1}\left\{\frac{(1-\delta) k^{n}[k(1+\beta)-(\alpha+\beta)]}{(1-\alpha) k(k+2-\delta)}\right\}^{1 / k+1}
$$

The result is sharp.

Proof: Let $f(z)$ is in $\sum_{p}(\alpha, \beta)$. Then by Theorem 2.1, we have

$$
\sum_{k=1}^{\infty} k^{n}[k(1+\beta)-(\alpha+\beta)]\left|a_{k}\right| \leq(1-\alpha)
$$

It is sufficient to show that

$$
\left|2+\frac{z f^{\prime \prime}(z)}{f^{\prime}(z)}\right| \leq(1-\delta)
$$

for $|z|<\gamma=\gamma(\alpha, \beta, \delta)$, where $\gamma(\alpha, \beta, \delta)$ is specified in the statement of the theorem. Then

$$
\left|2+\frac{z f^{\prime \prime}(z)}{f^{\prime}(z)}\right|=\left|\frac{\sum_{k=1}^{\infty} k(k+1) a_{k} z^{k-1}}{\frac{-1}{z^{2}}+\sum_{k=1}^{\infty} k a_{k} z^{k-1}}\right| \leq \frac{\sum_{k=1}^{\infty} k(k+1) a_{k}|z|^{k+1}}{1-\sum_{k=1}^{\infty} k a_{k}|z|^{k+1}}
$$

This will be bounded by $(1-\delta)$ if

$$
\sum_{k=1}^{\infty} \frac{k(k+2-\delta)}{1-\delta} a_{k}|z|^{k+1} \leq 1
$$

By (5.1), it follow that (5.2) is true if

$$
\frac{k(k+2-\delta)}{1-\delta}|z|^{k+1} \leq \frac{k^{n}[k(1+\beta)-(\alpha+\beta)]}{1-\alpha}, k \geq 1
$$




$$
|z| \leq\left\{\frac{(1-\delta) k^{n}[k(1+\beta)-(\alpha+\beta)]}{(1-\alpha) k(k+2-\delta)}\right\}^{1 / k+1}
$$

Setting $|z|=\gamma(\alpha, \beta, \delta)$ in (5.3), the result follows.

The result is sharp for the function

$$
f_{k}(z)=\frac{1}{z}+\frac{1-\alpha}{k^{n}[k(1+\beta)-(\alpha+\beta)]} z^{k},(k \geq 1) .
$$

\section{CLOSURE THEOREMS}

Let the functions $f_{j}(\mathrm{z})$ be defined, for $j=1,2, \ldots \ldots \ldots \ldots \ldots . . ., m$, by

$$
f_{j}(z)=\frac{1}{z}+\sum_{k=1}^{\infty} a_{k, j} z^{k}\left(a_{k, j} \geq 0\right)
$$

Theorem 6.1. Let $f_{j}(z) \in \sum_{p}(\alpha, \beta)(j=1,2, \ldots \ldots \ldots \ldots, m)$. Then the function

$$
h(z)=\frac{1}{z}+\sum_{k=1}^{\infty}\left(\frac{1}{m} \sum_{j=1}^{m} a_{k, j}\right) z^{k} .
$$

is in $\sum_{p}(\alpha, \beta)$.

Proof : Since $f_{j}(z) \in \sum_{p}(\alpha, \beta) \quad(j=1,2, \ldots \ldots \ldots ., m)$, is follows from Theorem 2.1 that

$$
\sum_{k=1}^{\infty} k^{n}[k(1+\beta)-(\alpha+\beta)] a_{k, j} \leq(1-\alpha),
$$

for every $\mathrm{j}=1,2$ …..........m. Hence,

$$
\begin{aligned}
& \sum_{k=1}^{\infty} k^{n}[k(1+\beta)-(\alpha+\beta)]\left(\frac{1}{m} \sum_{j=1}^{m} a_{k, j}\right) \\
& =\frac{1}{m} \sum_{j=1}^{m}\left(k^{n}[k(1+\beta)-(\alpha+\beta)] a_{k, j}\right) \leq 1-\alpha .
\end{aligned}
$$

From Theorem 2.1, if follows that $h(z) \in \sum_{p}(\alpha, \beta)$.

Theorem 6.2. The class $\Sigma_{p}(\alpha, \beta)$ is closed under convex linear combinations.

Proof: Let $f_{j}(z)(j=1,2)$ defined by (6.1) be in the class $\sum(\alpha, \beta)$. Then it is sufficient to show that

$$
h(z)=\mu f_{1}(z)+(1-\mu) f_{2}(z)(0 \leq \mu \leq 1)
$$

is in the class $\Sigma_{p}(\alpha, \beta)$, since

$$
h(z)=\frac{1}{z}+\sum_{k=1}^{\infty}\left[\begin{array}{ll}
\mu a_{k, 1} \\
+(1-\mu) a_{k, 2}
\end{array}\right] z^{k}
$$

then, we have from theorem 2.1 that

$$
\begin{aligned}
& \sum_{k=1}^{\infty} k^{n}[k(1+\beta)-(\alpha+\beta)]\left[\mu a_{k .1}+(1-\mu) a_{k, 2}\right] \\
& \leq \mu(1-\alpha)+(1-\mu)(1-\alpha) \\
& =1-\alpha \\
& \text { so, } h(z) \in \sum_{p}(\alpha, \beta) .
\end{aligned}
$$

Theorem 6.3. Let $f_{0}(z)=\frac{1}{z}$ and 


$$
f_{k}(z)=\frac{1}{z}+\frac{(1-\alpha)}{k^{n}[k(1+\beta)-(\alpha+\beta)]} z^{k}(k \geq 1)
$$

Then $\mathrm{f}(\mathrm{z})$ is in the class $\Sigma_{p}(\alpha, \beta)$ if and only if can be expressed in the from

$$
f(z)=\sum_{k=0}^{\infty} \mu_{k} f_{k}(z)
$$

where $\mu_{k} \geq 0$ and $\sum_{k=0}^{\infty} \mu_{k}=1$

Proof : Assume that

$$
f(z)=\sum_{k=0}^{\infty} \mu_{k} f_{k}(z)=\frac{1}{z}+\sum_{k=1}^{\infty} \frac{(1-\alpha)}{k^{n}[k(1+\beta)-(\alpha+\beta)]} \mu_{k} z^{k}
$$

Then it follows that

$$
\begin{aligned}
& \sum_{k=1}^{\infty} \frac{(1-\alpha)}{k^{n}[k(1+\beta)-(\alpha+\beta)]} \mu_{k} \frac{k^{n}[k(1+\beta)-(\alpha+\beta)]}{1-\alpha} \\
& \quad=\sum_{k=1}^{\infty} \mu_{k}=1-\mu_{0} \leq 1
\end{aligned}
$$

which implies that $f(z) \in \Sigma_{p}(\alpha, \beta)$.

Conversely, assume that the function $f(\mathrm{z})$ be in the class $\Sigma_{p}(\alpha, \beta)$.

Then $a_{k} \leq \frac{(1-\alpha)}{k^{n}[k(1+\beta)-(\alpha+\beta)]}$

Setting $\mu_{k}=\frac{k^{n}[k(1+\beta)-(\alpha+\beta)]}{(1-\alpha)} a_{k},\left(a_{k} \geq 1\right)$ and $\mu_{0}=1-\sum_{k=0}^{\infty} \mu_{k}$

we can see that $f(\mathrm{z})$ can be expressed in the form (6.6)

This completes the proof of theorem.

\section{MODIFIED HADAMARD PRODUCTS}

For $f_{j}(\mathrm{z})(j=1,2)$ defined by $(4.1)$, the modified Hadamard product of $f_{l}(\mathrm{z})$ and $f_{2}(\mathrm{z})$ is defined by

$$
\left(f_{1} * f_{2}\right)(z)=\frac{1}{z}+\sum_{k=1}^{\infty} a_{k, 1} a_{k, 2} z^{k}=\left(f_{2} * f_{1}\right)(z)
$$

Theorem 7.1: Let $f_{j}(z) \in \sum_{p}(\alpha, \beta)(j=1,2)$. Then, $\left(\mathrm{f}_{1} * \mathrm{f}_{2}\right)(\mathrm{z}) \in \sum_{p}(\xi, \beta)$,

The result is sharp for the function $\mathrm{f}_{\mathrm{j}}(\mathrm{z})(\mathrm{j}=1,2)$ given by

$$
\mathrm{f}_{\mathrm{j}}(\mathrm{z})=\frac{1}{z}+z \quad(\mathrm{j}=1,2)
$$

Proof : Using the technique for Schild and Silvarman [8]we need to find the largest

$$
\begin{aligned}
& \xi \text { such that } \\
& \sum_{k=1}^{\infty} \frac{k^{n}[k(1+\beta)-(\alpha+\beta)]}{1-\xi} a_{k, 1} a_{k, 2} \leq 1
\end{aligned}
$$

Since $f_{j}(z) \in \sum_{p}(\alpha, \beta)(j=1,2)$, we readly see that 
$\sum_{k=1}^{\infty} \frac{k^{n}[k(1+\beta)-(\alpha+\beta)]}{1-\alpha} a_{k, 1} \leq 1$

and

$\sum_{k=1}^{\infty} \frac{k^{n}[k(1+\beta)-(\alpha+\beta)]}{1-\alpha} a_{k, 2} \leq 1$

By the Cauchy Schwarz in equality we have

$$
\sum_{k=1}^{\infty} \frac{k^{n}[k(1+\beta)-(\alpha+\beta)]}{1-\alpha} \sqrt{a_{k, 1} a_{k, 2}} \leq 1
$$

This it is sufficient to show that

$$
\frac{k^{n}[k(1+\beta)-(\alpha+\beta)]}{1-\xi} a_{k, 1} a_{k, 2} \leq \frac{k^{n}[k(1+\beta)-(\alpha+\beta)]}{1-\alpha} \sqrt{a_{k, 1} a_{k, 2}}
$$

or equivalently, that

$$
\sqrt{a_{k, 1} a_{k, 2}} \leq \frac{1-\xi}{1-\alpha}
$$

Connecting with (7.7), it is sufficient to prove that

$$
\frac{1-\alpha}{k^{n}[k(1+\beta)-(\alpha+\beta)]} \leq \frac{1-\xi}{1-\alpha}
$$

It follows from (7.10) that

$$
\xi \leq 1-\frac{(1-\alpha)^{2}}{k^{n}[k(1+\beta)-(\alpha+\beta)]}
$$

Now defining the function $\phi(k)$ by

$$
\phi(k)=1-\frac{(1-\alpha)^{2}}{k^{n}[k(1+\beta)-(\alpha+\beta)]}
$$

We see that $\phi(k)$ is an increasing function of $\mathrm{k}(k \geq 1)$.

Therefore, we conclude that

$$
\xi \leq \phi(1)=\alpha
$$

which evidently completes the proof of theorem.

\section{CONCLUSION}

The work presented here is the generalization of some work done by earlier researchers. Further the research work can be continued by using the differential operator for the class and study the partial sums and integral operators also.

\section{REFERENCES}

[1] J.Clunie, On meromorphic schlicht functions, J. London Math.Soc. (34) (1959), 215-216.

[2] B.A.Frasin and M.Darus, On certain meromophic functions with positive coefficients, South East Asian Bull. Math. 28 (2004), 615623.

[3] O.P.Juneja and T. R. Reddy, Meromorphic starlike univalent functions with positive coefficients, Ann.Univ. Mariae Curie Sklodowska. Sect A 39, (1985), 65-76.

[4] J.E.Miller, Convex meromorphic mapping and related functions, Proc. Amer.Math. Soc. 25 (1970), 220-228

[5] M.L.Mogra, T.R. Reddy and O.P. Juneja, Meromorphic univalent functions with positive coefficients' Bull. Austral. Math. Soc. 32, (1985) 161-176.

[6] Ch.Pommerenke, On meromorphic starlike functions, Pacific J. Math. 13 (1963) 221-235

[7] W.C.Royster, Meromorphic starlike multivalent functions, Trans. Amer. Math. Soc.107 (1963). 300-308.

[8] A.Schild and H.Silverman,Convolutions of univalent functions with negative coefficients ,Ann.Univ.Mariea Curie-Sklodowska Sect,A 29 (1975) 99-107. 